\title{
Evaluation of coagulation parameters and liver enzymes among alcohol drinkers in Port Harcourt, Nigeria
}

This article was published in the following Dove Press journal:

International Journal of General Medicine

13 June 2013

Number of times this article has been viewed

\section{Teddy Charles Adias' \\ Everton Egerton ${ }^{2}$ \\ Osaro Erhabor ${ }^{3}$}

'Bayelsa College of Health Technology, Bayelsa State, Nigeria; ${ }^{2}$ Department of Medical Laboratory Science, Rivers State University of Science and Technology, Port Harcourt, Nigeria; ${ }^{3}$ Faculty of Medical Laboratory Science, Department of Haematology and Transfusion Medicine, Usmanu Danfodiyo University, Sokoto, Nigeria
Correspondence: Osaro Erhabor Usmanu Danfoditio University, Sokoto, Nigeria

Email n_osaro@yahoo.com
Abstract: Alcohol is a major contributor to the global burden of disease, disability, and death in high, middle, and low-income countries. Harmful use of alcohol is one of the main factors contributing to premature deaths and avoidable disease burden worldwide and has a major impact on public health. The aim of this present cross-sectional study was to investigate the effect of alcohol consumption on coagulation parameters and liver enzymes of subjects in Port Harcourt, Nigeria. Two hundred adults consisting of 120 alcohol dependent subjects and 80 age, gender-matched nondrinkers aged 25-65 years (mean age $45.25 \pm 11.50$ years) were enrolled in this study. Of the 120 chronic alcohol drinkers, 37 were dependent on local dry gin, while 83 were dependent on other alcoholic beverages. The mean values of the liver enzymes, aspartate aminotransferase and gamma glutamyl transferase, were significantly higher $(P=0.002$ and $P=0.02$ respectively) among the chronic alcohol consumers compared with their nondrinker counterparts. Although the value of alanine aminotransferase was higher in the chronic drinkers, it did not reveal any significant difference $(P=0.11)$. The coagulation parameters, prothrombin time and activated partial thromboplastin time were investigated among chronic drinkers and nondrinkers. The mean value of prothrombin time and activated partial thromboplastin time was significantly higher in the chronic alcohol drinkers compared to the nondrinkers ( $P=0.04$ and $P=0.02$ respectively). We observed a positive and significant correlation between values of liver enzymes, serum gamma glutamyl transferase and aspartate aminotransferase, and values of prothrombin time among alcohol consumers ( $r=0.72$ and $r=0.68$ respectively). The implementation of policies to target harm reduction strategies among alcoholics is urgently needed, alongside the building of a strong base of public awareness and community support required for the continuity and sustainability of alcohol policies. There is also the need for the Nigerian government to enforce tighter regulations and restrictions on the production and distribution of alcoholic beverages to reduce harmful use, and protect young people and other vulnerable groups.

Keywords: coagulation parameters, liver enzymes, chronic alcoholics, Port Harcourt, Nigeria

\section{Introduction}

Harmful use of alcohol is one of the main factors contributing to premature deaths and avoidable disease burden worldwide and has a major impact on public health. Harmful use of alcohol was estimated to cause about 2.3 million premature deaths worldwide in 2009 (3.7\% of global mortality and $4.4 \%$ of the global burden of disease). ${ }^{1}$ Alcoholic beverages, and the problems they engender, have been familiar fixtures in human societies. It is likely that alcohol use and related disorders will increase as a public health problem in Nigeria over the coming years and the global burden of ill health related to alcohol use will grow. The patterns of alcohol intake around the 
world are constantly evolving, and alcohol is ubiquitous today. Alcohol is a major contributor to the global burden of disease, disability, and death in high, middle, and low-income populations. ${ }^{1}$ It is estimated that $3.5 \%$ of the global burden of disease is attributable to alcohol. ${ }^{2-3}$ Alcohol consumption has been linked to more than 60 medical conditions and is also linked to categories of disease whose relative impact on the global burden is predicted to increase. ${ }^{4}$

Alcoholism has been defined as an individual's dependence on alcohol, and alcohol misuse or uncontrolled drinking habit, which adversely interferes with biological, social, and mental well-being. ${ }^{5}$ Alcohol-related problems are a major public health issue. A review of the studies on alcohol use in Nigeria shows that there has been a rapid increase in alcohol availability and consumption in recent times particularly among young adults. ${ }^{5}$ Studies in Nigeria have focused on lifetime and current prevalence rates of alcohol use $^{6}$ without exploring alcohol-related health problems, particularly concerned with the hemostatic parameters and liver function.

Alcohol consumption is a major factor in the global burden of disease and should be considered a public health priority globally, regionally, and nationally for the vast majority of countries in the world..$^{7-9}$ The potential effect of alcohol consumption on the hemostatic system and liver function among chronic alcohol consumers in Port Harcourt, Nigeria is not known. The aim of this present cross-sectional study was to investigate the effect of alcohol consumption on liver enzymes and coagulation parameters of subjects in Port Harcourt, Nigeria as a way of generating scientific evidence to enable the strategic interventions that can help prevent or minimize alcoholrelated harm in Nigeria.

\section{Materials and methods Subjects}

This prospective case-control study included 200 adults ( $\geq 18$ years) age range (25-65 years) and mean age $(45.25 \pm 11.50$ years). There were 120 alcohol dependent (subjects), and 80 age, gender-matched nondrinkers (controls). The aim of this study was to investigate the effect of chronic alcohol consumption on liver enzymes; aspartate aminotransferase (AST), alanine aminotransferase (ALT), and gamma glutamyl transferase (GGT) and some hemostatic parameters; prothrombin time (PT) and activated partial thromboplastin time (APTT), of alcohol dependent subjects and nondrinkers. The values were compared statistically for any significant difference. Confirmation of alcoholism was established on the basis of the Short Michigan Alcoholism Screening Test (SMAST). ${ }^{10}$ The SMAST is a 13-item, self-reporting and effective diagnostic instrument developed from the Michigan Alcoholism Screening Test (MAST) questionnaire. It has better sensitivity and diagnostic accuracy for chronic alcohol consumption, and thus does not have the tendency for false positive results as does the MAST. ${ }^{11}$ Subjects for this crosssectional study were all out-patients selected based on a random-cluster sample of different suburban communities (Elechi beach, Ogu, Okujagu, Duck yard, and Rex Lawson waterfront) in Port Harcourt within the period of 12 months. Written informed consent was obtained from all subjects and control individuals. Ethical approval was obtained from the research and ethics committee in the Department of Medical Laboratory Science of the Rivers State University of Science and Technology, Port Harcourt, Nigeria. Subjects were included according to these criteria: age $\geq 18$ years, no history of present or past 2 weeks' medication use prior to the collection of samples, and willingness to give written informed consent after discussion of study procedures. Exclusion criteria included: pregnancy, age $<18$ years, history of hematological diseases, malignancies or infections, chronic illnesses such as tuberculosis, and diabetes mellitus. Alcohol dependent subjects in this study were individuals who drink alcoholic beverages at least once daily. Duration of alcohol intake among the subjects ranged from 3-30 years. Controls included age and gender-matched nondrinkers with neither current nor past history of regular alcohol drinking.

\section{Methods}

Eight milliliters of blood sample was drawn aseptically using the S-Monovette vacutainer blood collection system (Sarstedt, Nümbrecht, Germany) from the median antecubital vein for all the subjects and control participants. Three milliliters of blood was collected into a citrate bottle, while $5 \mathrm{~mL}$ was collected into a gel tube without anticoagulant. The gel sample was allowed to clot, centrifuged lightly and the serum separated. The serum sample was used for the manual colorimetric determination of liver enzymes AST, ALT, and GGT using Randox (Crumlin, UK) test kits. The citrate samples were centrifuged for 5 minutes at $1000 \mathrm{rpm}$ to obtain the citrated plasma that was used to determine PT and APTT using standard manual laboratory methods within 4 hours of sample collection. Test procedures were conducted according to the instructions in the manufacturer's standard operating manual. 


\section{Statistical analysis}

Data were entered and analyzed using statistical package SPSS version 9 (SPSS Inc, Chicago, IL, USA). Statistical analysis included descriptive analysis of mean, standard deviation student $t$-test and Chi-square analysis. A $P$-value of $>0.05$ was considered to be statistically significant in all statistical analyses. Correlation was compared using a version of linear regression analysis.

\section{Results}

Two hundred adults (120 alcoholics and 80 age and gender matched nondrinkers) aged 25-65 years (mean age $45.25 \pm 11.50$ ) were enrolled in this study. Of the 120 chronic alcohol drinkers, 37 were dependent on local dry gin while 83 were dependent on other alcoholic beverages. Duration of alcohol intake among the subjects ranged from 3-30 years as shown in Figure 1.

\section{Liver enzymes in chronic alcohol drinkers versus nondrinkers}

The mean values of AST and GGT were significantly higher ( $P=0.002$ and $P=0.02$ respectively) among the chronic alcohol consumers compared with their nondrinking counterparts. Though the value of ALT was higher in the chronic alcohol drinkers, it did not reach statistical significance $(P=0.11)$. Table 1 shows the mean values of these liver enzymes, AST, ALT, and GGT, in chronic alcohol drinkers and nondrinkers. We observed a positive and significant correlation between values of liver enzymes, GGT and AST, and values of PT among chronic alcohol drinkers ( $r=0.72$ and $r=0.68$ respectively).

\section{Comparison of values of coagulation parameters in chronic alcohol drinkers and nondrinkers}

The coagulation parameters, PT and APTT, were investigated among chronic alcoholics and nondrinkers. The mean

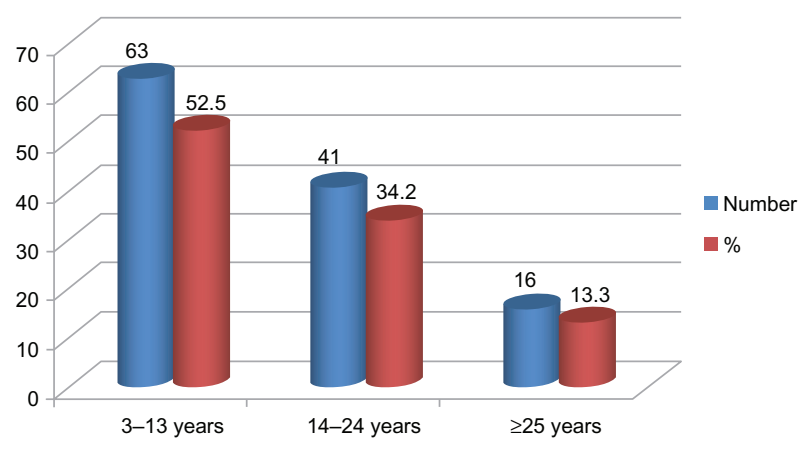

Figure I Distribution of subjects based on duration of alcohol intake.
Table I Comparison of values of liver enzymes in chronic alcohol drinkers and nondrinkers

\begin{tabular}{llll}
\hline $\begin{array}{llll}\text { Liver enzyme } \\
\text { parameters }\end{array}$ & $\begin{array}{l}\text { Mean } \pm \text { SD } \\
\text { alcohol drinkers }\end{array}$ & P-value \\
\cline { 2 - 3 } & $\begin{array}{l}\text { Chronic } \\
\text { alcondrinkers }\end{array}$ & \\
\hline AST & $23.4 \pm 19.7$ & $11.0 \pm 8.4$ & 0.002 \\
ALT & $10.5 \pm 7.7$ & $8.3 \pm 5.8$ & 0.11 \\
GGT & $42.3 \pm 23.8$ & $31.8 \pm 19.5$ & 0.02 \\
\hline
\end{tabular}

Abbreviations: ALT, alanine aminotransferase; AST, aspartate aminotransferase; GGT, gamma glutamyl transferase; SD, standard deviation.

values of PT and APTT were significantly higher among chronic alcoholics compared to the nondrinkers $(P=0.04$ and $P=0.02$ respectively). Table 2 shows the comparison between the coagulation parameters of alcoholics and nondrinkers.

\section{Discussion}

Alcoholism is one of the numerous widespread noncommunicable diseases that is ravaging the human race and poses a serious threat to the global health sector. ${ }^{12,13}$ This present cross-sectional study has investigated the effects of chronic alcohol consumption on the liver enzymes and coagulation parameters in Port Harcourt, Nigeria.

Liver enzymes such as AST, ALT, and GGT, which remain vital indicators of hepatocellular injuries possibly due to long-term alcohol dependence, were considered in this study. Except for the mean value of the ALT (10.5 \pm 7.7$)$, which did not show any significant change $(P>0.05)$, the mean values of the AST $(23.4 \pm 19.7)$ and GGT $(42.3 \pm 23.8)$ showed a dramatic rise among the chronic alcohol drinking subjects compared with their nondrinking counterparts $(P<0.05)$. These increases in the various enzymes are consistent with previous reports which found the liver as the prime target for alcohol induced diseases. ${ }^{14,15}$ Similarly, a previous report in Ghana among 60 total abstainers, 56 social drinkers, and 100 alcoholics indicated that GGT and AST are sufficiently sensitive to detect chronic alcoholics and that the serum GGT and AST showed progressive rise with increasing alcohol intake. ${ }^{16}$

Table 2 Comparison between the coagulation parameters of alcohol drinkers and nondrinkers

\begin{tabular}{llll}
\hline $\begin{array}{l}\text { Coagulation } \\
\text { parameters }\end{array}$ & Mean \pm SD & P-value \\
\cline { 2 - 3 } & Alcohol drinkers & Nondrinkers & \\
\hline PT (seconds) & $20.7 \pm 3.1$ & $15.5 \pm 3.7$ & 0.04 \\
APTT (seconds) & $38.1 \pm 6.7$ & $33.5 \pm 5.3$ & 0.02 \\
\hline
\end{tabular}

Abbreviations: APTT, activated partial thromboplastin time; PT, prothrombin time; $\mathrm{SD}$, standard deviation. 
GGT activity in serum is useful in the assessment of alcohol-induced liver disease and for demonstrating to patients the toxic effects of their drinking habits on the liver. Serial measurements of GGT activity in serum have proved a valuable marker in monitoring the progress of therapy as well as alleged abstention from alcohol in the known alcoholic. ${ }^{17}$ A previous report ${ }^{18}$ involving 14 moderate drinkers who abstained from alcohol for 4 weeks indicated that the activity of GGT in their serum showed a large decrease. However immediately after the period of abstention, an introduction of ethanol challenge of $1 \mathrm{~g} / \mathrm{kg}$ produced a marked increase in serum GGT at 24 hours, followed by a slow decline thereafter. AST activity in serum was significantly increased at 24 hours while ALT showed much smaller or no change. Elevated levels of serum enzymes are frequently associated with alcohol-related liver diseases. However a major limitation of the use of the liver enzymes parameter in the early detection of alcoholism, as well as in the diagnosis of alcoholrelated diseases, has been the fact that the sensitivities and specificities of these liver enzyme markers vary considerably. They may be influenced by nonalcohol related diseases, the effect of enzyme-inducing drugs, nutritional factors, metabolic disorders, age, and smoking. Consequently there is neither a sensitive and specific single laboratory enzyme marker, nor a test combination that is reliable enough for the exact diagnosis between alcohol and nonalcohol related liver damage. GGT is the most widely used laboratory marker of alcoholism and heavy drinking. In these patients, GGT serum levels can be markedly altered $(>10$ times the upper reference value). GGT together with other biochemical abnormalities (AST/ALT ratio $>2$ ) detects $34 \%-85 \%$ of problem drinkers and alcoholics. ${ }^{19}$ However, the non-specificity of increased serum GGT limits its use for general screening purposes. An elevated level of serum AST and ALT in an alcoholic or a heavy consumer of alcohol indicates alcohol-induced organ damage. The use of test combinations significantly improves the information received with single serum enzyme determinations. An AST/ALT ratio greater than 2.0 can be considered as highly suggestive for the alcoholic etiology of the liver injury. ${ }^{20}$ Better discrimination between alcoholic and nonalcoholic origin of liver disease may be achieved by the determination of the ratio of GGT to alkaline phosphatase. If this ratio exceeds $1: 4$ the specificity of the finding in favor of alcoholic liver injury is $78 \%$.

We investigated the coagulation parameters among chronic alcohol drinkers and nondrinkers. The mean values of the PT $(20.7 \pm 3.1)$ and APTT $(38.1 \pm 6.7)$ were significantly higher among the chronic alcohol drinkers compared to the nondrinkers $(15.5 \pm 3.7 ; 33.5 \pm 5.3$; respectively). The PT and its derived measure, the International Normalised Ratio (INR), test for factors such as FII, FV, FVII, FX and fibrinogen. The APTT is considered a good screening test for deficiencies of FVIII, FIX, FXI, and FXII. Coagulation factors are synthesized in the liver. Alcohol consumption seems to have a negative effect on the liver with attendant negative impact on the synthesis of coagulation factors. Our finding in this present study is consistent with previous reports which indicated that alcoholic hepatitis and alcohol-associated liver cirrhosis are the eminent causes of decreased production of blood clotting factors. ${ }^{21-24}$ Ethanol has multiple effects on hemostasis, affecting coagulation factors, and the fibrinolytic system. Although conflicting results exist in the literature, growing evidence is pointing to the following effects of ethanol or its metabolites: decreased levels of fibrinogen and selected other coagulation factors, and increased fibrinolysis. Ethanol intoxication is a common contributor to traumatic injury. There is controversy as to whether ethanol consumption contributes to the coagulation differences seen between men and women after trauma. A recent report indicates that consumption of commonly ingested quantities of alcohol correlated with the development of a hypocoagulable state in men but had no effect on coagulation status in women. This phenomenon may contribute to differences in post-trauma coagulation status previously noted between genders. ${ }^{25}$

We observed that values of liver enzymes (serum GGT and AST) were positively correlated with values of coagulation parameter (PT) among chronic alcohol consumers ( $r=0.72$ and $r=0.68$ respectively). This finding is an indication that raised values of coagulation parameters were directly proportional to raised liver enzymes. This finding is consistent with previous reports ${ }^{21-25}$ which indicated that alcoholic hepatitis and liver cirrhosis-related elevation in liver enzymes are associated with alcohol-dependent decrease in the production of blood clotting factors and derangement in coagulation parameters.

The liver plays a central role in the clotting process, and acute and chronic liver diseases are invariably associated with coagulation disorders due to multiple causes: decreased synthesis of clotting and inhibitor factors, decreased clearance of activated factors, quantitative and qualitative platelet defects, hyperfibrinolysis, and accelerated intravascular coagulation. ${ }^{26}$ Patients with liver disease, have a disturbed balance of pro-coagulant and anti-coagulant factors deviating from the normal coagulation cascade..$^{23,27}$ 
There is evidence linking coagulation parameters and liver enzymes to clinical outcomes. The liver is responsible for the production of coagulation factors. The INR measures the speed of a particular pathway of coagulation, comparing it to normal. Increased levels of INR mean that blood is taking more time than usual to coagulate or clot. The INR will be increased only if the liver is so damaged that synthesis of vitamin K-dependent coagulation factors has been impaired; it is not a sensitive measure of liver function. PT and its derived measure INR are measures of the extrinsic pathway of coagulation. PT and INR are used to determine the clotting tendency of blood, in the measure of liver damage and vitamin $\mathrm{K}$ status. AST is similar to ALT in that it is another enzyme associated with liver parenchyma. It is raised in acute liver damage, but is also present in red blood cells, and cardiac and skeletal muscle and is therefore not specific to the liver. The ratio of AST to ALT is clinically useful in differentiating between causes of liver damage. Elevated AST level is not specific for liver damage; it has also been used as a cardiac marker. AST/ ALT elevations are a clinical indication of liver cell necrosis. When the levels of AST and ALT are greater than three times normal but not $>1000$ IU/L, the effects can include alcohol toxicity, viral hepatitis, drug induced liver cancer, sepsis, Wilson disease, post-transport rejection of liver, autoimmune hepatitis, and steatohepatitis (nonalcoholic). ${ }^{28}$ GGT although reasonably specific to the liver and a more sensitive marker for cholestatic damage, can be elevated with even minor, subclinical levels of liver dysfunction. It is raised in chronic alcohol toxicity.

Alcohol dependence is a fast growing public health problem worldwide. The reasons for habitual and increasing alcohol dependence have been linked to: gaining courage in risk taking behaviors; overcoming feelings of inferiority and rebelling against authorities; lack of confidence and strong feelings of low self-esteem; and its use as medicine, sedatives, and anesthetics in the hostile world of cold and hunger, religious/tribal life styles among others. ${ }^{29}$ Other predisposing factors include: genetically determined physical reactions to alcohol that increase the likelihood that some individuals can drink alcohol in large enough quantities to become chronic alcoholics; ${ }^{30}$ influence of biological relatives who are chronic alcoholics. ${ }^{31}$ Alcohol dependence is a major socioeconomic problem, associated with severe effects on public health with about $10 \%-30 \%$ of all patients admitted into tertiary and secondary health institutions and a significant percentage of psychiatric admissions being victims. ${ }^{32}$

\section{Conclusion}

There are several social problems arising from alcoholism, caused by the pathological changes in the brain and the intoxicating effects of alcohol. ${ }^{33-37}$ The implementation of policies on which to base harm reduction strategies is urgently needed. There is a need for increased policies and public health programs to reduce alcohol related effects on liver enzymes and coagulation parameters among chronic alcoholics. ${ }^{38-40}$ There is a need to build a strong base of public awareness and community support to help ensure the continuity and sustainability of alcohol policies. The Nigerian government needs to enforce tighter regulations and restrictions (pricing, taxation, age of consumers, the type of retail establishments that can sell alcoholic beverages, licensing, limits on hours and days of sale, and regulations on vendors and the density of outlets) on the production and distribution of alcoholic beverages to reduce harmful use as well as protect young people and other vulnerable groups.

\section{Disclosure}

The authors report no conflicts of interest in this work.

\section{References}

1. Alcohol and Public Policy Group. Alcohol: no ordinary commodity - a summary of the second edition. Addiction. 2010;105:769-779.

2. Rehm J, Rehn N, Room R, et al. The global distribution of average volume of alcohol consumption and patterns of drinking. Eur Addict Res. 2003;9:147-156.

3. Das SK, Balakrishnan V, Vasudevan DM. Alcohol: its health and social impact in India. Natl Med J India. 2006;19:94-99.

4. Das SK, Mukherjee S, Vasudevan DM. Non-alcoholic fatty liver diseases: an underrecognized cause with emerging importance. Curr Sci. 2006;90:659-665.

5. Ceccanti M, Sasso GF, Nocente R, et al. Hypertension in early alcohol withdrawal in chronic alcoholics. Alcohol. 2006;41:5-10.

6. Abiodun OA. Drug abuse and its clinical implications with special reference to Nigeria. Centr Afr J Med. 1991;37:24-30.

7. Adelekan ML, Abiodun OA, Obayan AO, Oni G, Ogunremi OO. Prevalence and pattern of substance use among undergraduates in a Nigerian University. Drug Alcohol Depend. 1992;29:255-261.

8. Room R, Graham K, Rehm J, Jernigan D, Monteiro M. Drinking and its burden in a global perspective: policy considerations and options. Eur Addict Res. 2003;9:165-175.

9. Rehm J, Room R, Graham K, Monteiro M, Gmel G, Sempos CT. The relationship of average volume of alcohol consumption and patterns of drinking to burden of disease: an overview. Addiction. 2003;98:1209-1228.

10. Harburg E, Gunn R, Gleiberman L, Roeper P, DiFranceisco W, Caplan R. Using the Short Michigan Alcoholism Screening Test to study social drinkers: Tecumseh, Michigan. J Stud Alcohol. 1988;49(6):522-531.

11. Hirata ES, Almeida OP, Funari RR, Klein EL. Validity of the Michigan Alcoholism Screening Test (MAST) for the detection of alcohol-related problems among male geriatric outpatients. Am J Geriatr Psychiatry. 2001;9(1):30-34.

12. Vaswani M, Rao RV. Biological measures in the diagnosis of alcohol dependence using discriminant analysis. Indian J Med Sci. 2005;59:423-430. 
13. Ceccanti M, Sasso GF, Nocente R, et al. Hypertension in early alcohol withdrawal in chronic alcoholics. Alcohol unit, University La Sapienza, Rome. Instituto Superiore di Sanita, Rome and Emergency Department, University La Sapienza, Rome, Italy. 2005:1-8.

14. Teschke RZ. Alcohol and gamma-lutamyltransferase. Gastroenterol. 1993;31(Suppl 5):49-52.

15. Whitfield JB, Hensley WJ, Bryden D, Gallagher H. Some laboratory correlates of drinking habits. Ann Clin Biochem. 1978;15:297-303.

16. Quaye IK, Nyame PK, Dodoo D, Gyan B, Adjei AA. Biochemical and haematological markers of alcohol intake in Ghanaians. West Afr J Med. 1992;11:199-202.

17. Boone DJ, Tietz NW, Weinstock A. Significance of gamma-glutamyl transferase (GGT) activity measurements in alcohol-induced hepatic injury. Ann Clin Lab Sci. 1977;7:25-28.

18. Nemesánszky E, Lott JA, Arato M. Changes in serum enzymes in moderate drinkers after an alcohol challenge. Clin Chem. 1988;34: 525-527.

19. Salaspuro M. Use of enzymes for the diagnosis of alcohol-related organ damage. Enzyme. 1987;37:87-107.

20. Cohen JA, Kaplan MM. The SGOT/SGPT ratio - an indicator of alcoholic liver disease. Dig Dis Sci. 1979;24:835-838.

21. Dimmitt SB, Rakic V, Puddey IB, et al. The effects of alcohol on coagulation and fibrinolytic factors: a controlled trial. Blood Coagul Fibrinolysis. 1998;9:39-45.

22. Ragni MV, Lewis JH, Spero JA, Hasiba U. Bleeding and coagulation abnormalities in alcoholic cirrhotic liver disease. Alcohol Clin Exp Res. 1982;6:267-274.

23. Tripodi A, Mannucci PM. The coagulopathy of chronic liver disease. N Engl J Med. 2011;365:147.

24. Caldwell SH, Hoffman M, Lisman T, et al. Coagulation disorders and hemostasis in liver disease: pathophysiology and critical assessment of current management. Hepatology. 2006;44:1039.

25. Spoerke N, Underwood S, Differding J, et al. Effects of ethanol intoxication and gender on blood coagulation. J Trauma. 2010;68: 1106-1111.

26. Amitrano L, Guardascione MA, Brancaccio V, Balzano A. Coagulation disorders in liver disease. Semin Liver Dis. 2002;22:83-96.
27. Tripodi A, Anstee QM, Sogaard KK, et al. Hypercoagulability in cirrhosis: causes and consequences. J Thromb Haemost. 2011;9:1713.

28. Siddiqi AI, Siddiqeh M, Mehmood A, Siddiqui AM. Alanine aminotransferase/aspartate aminotransferase ratio reversal and prolonged prothrombin time: a specific indicator of hepatic cirrhosis. J Ayub Med Coll Abbottabad. 2007;19:22-24.

29. Lee EE. Alcohol, Proof of What? New York: Simon and Schuster; 1977.

30. Fantozzi R, Caramelli L, Ledda F, et al. Biological markers and therapeutic outcome in alcoholic disease: a twelve-year survey. Klin Wochenschr. 1987;65:27-33.

31. Rivara FP, Garrison MM, Ebel B. Mortality attributable to harmful drinking in the United States, 2000. J Stud Alcohol. 2004;65: 530-536.

32. Latvala J, Parkkila S, Niemela O. Excess alcohol consumption is common in patients with cytopenia: studies in blood and bone marrow cells. Alcohol Clin Exp Res. 2004;28:619-924.

33. McCully C. Goodbye Mr Wonderful. Alcohol, Addiction and Early Recovery. London: Jessica Kingsley Publishers. 2004.

34. Isralowitz R. Drug Use: A Reference Handbook. Santa Barbara, California.

35. Langdana FK. Macroeconomic Policy: Demystifying Monentary and Fiscal Policy, 2nd ed. New York: Springer; 2009.

36. Gifford M. Alcoholism (Biographies of Disease). Santa Barbara, California: Greenwood Press; 2010:89-91.

37. Schadé JP. The Complete Encyclopedia of Medicine and Health. Yardville, NJ: Foreign Media Books; 2006.

38. Giannini EG, Testa R, Savarino V. Liver enzyme alteration: a guide for clinicians. Pricing and taxation, regulating the physical availability of alcohol, modifying the drinking context, drink-driving countermeasures, restrictions on marketing, education and persuasion strategies, and treatment and early intervention services. CMAJ. 2005;172: 367-379.

39. Cochrane J, Chen H, Conigrave KM, Hao W. Alcohol use in China. Alcohol Alcohol. 2003;38:537-542.

40. Das SK, Balakrishnan V, Vasudevan DM. Alcohol: its health and social impact in India. Natl Med J India. 2006;19:94-99.
International Journal of General Medicine

\section{Publish your work in this journal}

The International Journal of General Medicine is an international, peer-reviewed open-access journal that focuses on general and internal medicine, pathogenesis, epidemiology, diagnosis, monitoring and treatment protocols. The journal is characterized by the rapid reporting of reviews, original research and clinical studies across all disease areas.

\section{Dovepress}

A key focus is the elucidation of disease processes and management protocols resulting in improved outcomes for the patient.The manuscript management system is completely online and includes a very quick and fair peer-review system. Visit http://www.dovepress.com/ testimonials.php to read real quotes from published authors. 